\title{
DARK ENERGY WITHOUT DARK ENERGY*
}

\author{
David L. Wiltshire ${ }^{\dagger}$ \\ Department of Physics and Astronomy, University of Canterbury, \\ Private Bag 4800, Christchurch 8140, New Zealand \\ ${ }^{\dagger}$ E-mail: David.Wiltshire@canterbury.ac.nz \\ http://www2.phys.canterbury.ac.nz/ dlw24/
}

\begin{abstract}
An overview is presented of a recently proposed "radically conservative" solution to the problem of dark energy in cosmology. The proposal yields a model universe which appears to be quantitatively viable, in terms of its fit to supernovae luminosity distances, the angular scale of the sound horizon in the cosmic microwave background (CMB) anisotropy spectrum, and the baryon acoustic oscillation scale. It may simultaneously resolve key anomalies relating to primordial lithium abundances, CMB ellipticity, the expansion age of the universe and the Hubble bubble feature. The model uses only general relativity, and matter obeying the strong energy condition, but revisits operational issues in interpreting average measurements in our presently inhomogeneous universe, from first principles. The present overview examines both the foundational issues concerning the definition of gravitational energy in a dynamically expanding space, the quantitative predictions of the new model and its bestfit cosmological parameters, and the prospects for an era of new observational tests in cosmology.
\end{abstract}

Keywords: dark energy, theoretical cosmology, observational cosmology

\section{Introduction}

Dark energy is widely described as the biggest problem in cosmology today; one which may demand new physics and a theoretical paradigm shift. ${ }^{1}$ In this paper I suggest that the solution to the problem of dark energy is intimately related to the correct understanding of observational anomalies - in particular, the observed abundance and emptiness of voids, which has also elicited separate calls for a paradigm shift. ${ }^{2}$ I propose that the paradigm shift that is required to understand both these issues actually entails no "new" physics, but a revisiting of fundamental issues relating to the sub-

*Based on talks presented at the NZIP2007, GRG18 and Dark2007 conferences. 
tlety of the definition of gravitational energy within general relativity, and its relation to the careful modelling of the distribution of matter that we actually observe.

The punchline is that cosmic acceleration can be understood as an apparent effect, and dark energy as a misidentification of those aspects of cosmological gravitational energy which by virtue of the strong equivalence principle cannot be localized, ${ }^{3}$ namely gradients in the quasilocal gravitational energy associated with spatial curvature gradients, and the kinetic energy of expansion, between bound systems and the volume-average position in freely expanding space. With this interpretation, a two-scale model can be constructed, ${ }^{3}$ and a simple exact solution ${ }^{4}$ of the relevant equations of cosmic evolution, the Buchert equations, ${ }^{5}$ can be found. This solution provides a fit to type Ia supernovae (SneIa) luminosity distances which is statistically indistinguishable from that of the standard Lambda Cold Dark Matter $(\Lambda \mathrm{CDM})$ model, while simultaneously satisfying key independent cosmological tests and offering the potential to resolve significant observational anomalies. ${ }^{6}$

Over the past decade we have come to think of "dark energy" as a homogeneous isotropic fluid-like quantity, with a local pressure related to its energy density by $P=w \rho$, where $w<-1 / 3$, so that the strong energy condition is violated. In fact, observations appear to be most consistent with a pure vacuum energy, $w=-1$, and determination of the value of the parameter, $w(z)$, as a function of redshift, $z$, is seen as the goal of "precision cosmology". What is proposed here, if correct, will turn this situation on its head. We can look forward to an era of precision cosmology, but one in which the focus will be on the complex hierarchical structure of the universe, rather than any one simple fluid equation of state. Nonetheless, while the proposed solution is intimately connected to the growth of inhomogeneities, and their backreaction on the geometry of the universe, ${ }^{7}$ at its heart it addresses the question of the normalization of gravitational energy relative to observers within the observed structure. Thus I claim that the solution to the central foundational question does concern energy, and since "nothing" is "dark" the terminology "dark energy" is actually quite apt for the new proposal, if the community will allow the liberty of a change to the assumed definition of those words.

In this paper I will give an overview of the proposed solution to the problem of dark energy, the extent of its present quantitative successes, and more importantly the directions for future work. I will present fewer technical details than may be found in papers already published ${ }^{3,4,6}$ or in 
preparation. ${ }^{8,9}$ I aim to give a general overview to researchers in astrophysics, particle physics or general relativity, who have no specific prior experience with the averaging of inhomogeneous cosmological models.

\section{The universe we observe}

Our most widely tested "concordance model" of the universe relies on the assumption of an isotropic homogeneous geometry at all epochs of cosmic evolution. By the evidence of the cosmic microwave background (CMB) radiation, the universe was very smooth at the time of last scattering, and these assumptions were completely well-justified then. The departure from homogeneity was of order $\delta \rho / \rho \sim 10^{-5}$ in photons and the baryons that couple to them, and perhaps of order $\delta \rho / \rho \sim 10^{-3}$ in non-baryonic dark matter, which gives rise to the potential wells responsible for the dominant Sachs-Wolfe effect. By the Copernican principle, the assumption of global isotropy and homogeneity is completely justified at the epoch of last scattering, and it is safe to assume that the evolution of the universe was therefore extremely closely modelled by the Friedmann-Lemaître-Robertson-Walker (FLRW) solutions at that epoch. Furthermore, if we consider the spectrum of CMB anisotropies ${ }^{10,11}$ then overall it appears that globally the universe is very close to spatially flat. Its initial evolution at the time of last scattering would therefore have been very close to that of an Einstein-de Sitter model at that epoch, even in the case of the $\Lambda \mathrm{CDM}$ paradigm since "dark energy" only becomes appreciable at late epochs.

At the present epoch, however, the distribution of matter is far from homogeneous on scales less than 150-300 Mpc. The actual universe has a sponge-like structure, dominated by huge voids. These voids are surrounded by bubble walls, and threaded by filaments, within which clusters of galaxies are located. Recent surveys suggest ${ }^{12}$ that some $40-50 \%$ of the present volume of the universe is in voids of a characteristic scale $30 h^{-1} \mathrm{Mpc}$, where $h$ is the dimensionless Hubble parameter, $H_{0}=100 h \mathrm{~km} \mathrm{sec}^{-1} \mathrm{Mpc}^{-1}$. If smaller minivoids ${ }^{13}$ and larger supervoids ${ }^{14}$ are included, then our observed universe is presently void-dominated by volume.

Quite apart from the fact that this observed structure appears emptier than the vistas that Newtonian $N$-body simulations typically produce, the mere fact that the universe is presently inhomogeneous means that the assumptions implicit in the FLRW approximation can no longer be justified at the present epoch in the almost exact sense that they were justified at the epoch of last scattering. Homogeneity only applies at the present day in an average sense. The manner in which we take the average, and the 


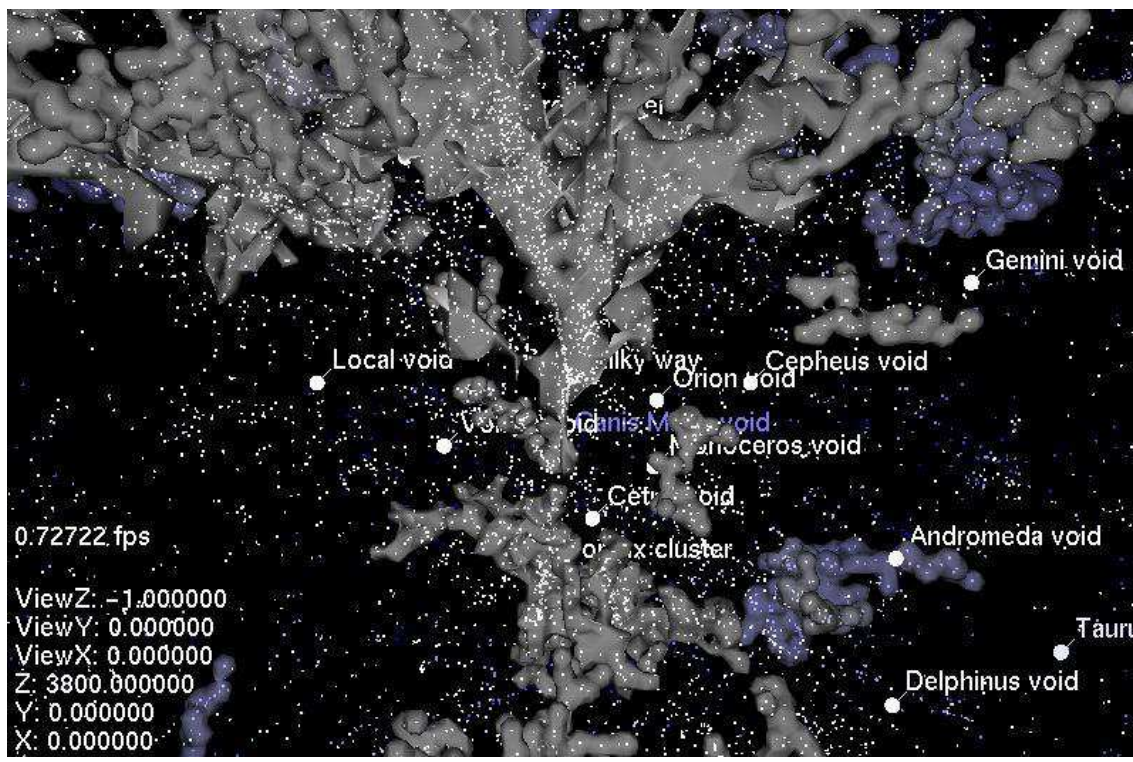

Fig. 1. Local voids and bubbles from the 6df survey. Courtesy of A. Fairall.

operational issues associated with this are not trivial, since the problem of fitting the local geometry of bound systems to the full dynamical geometry of the evolving universe ${ }^{15,16}$ is a complicated one.

Since a broadly isotropic Hubble flow is observed, an almost FLRW geometry would appear to be a good approximation at some level of averaging, if our position is a typical one. I will demonstrate, however, that the assumption that the observed universe evolves exactly as a smooth featureless dust fluid means that we factor out central physically important questions which need to be understood to correctly relate our own observations to the average geometry.

\subsection{The Sandage-de Vaucouleurs paradox}

There is a central foundational paradox concerning the expansion of the universe, which others have called the "Hubble-de Vaucouleurs paradox"17,18 or the "Hubble-Sandage paradox" ${ }^{19}$ but which I will call the Sandage-de Vaucouleurs paradox since it was originally raised by Sandage and collaborators $^{20}$ in objection to de Vaucouleurs' hierarchical cosmology. ${ }^{21}$

The problem is that in the conventional way of thinking about cosmological averages, below the scale of homogeneity we should expect large 
statistical scatter in the peculiar velocities of galaxies. In fact, if we were to average on scales of order $20 \mathrm{Mpc}$, which is about $10 \%$ of the scale of homogeneity, then the statistical scatter should be so large that no linear Hubble law can be extracted. Yet $20 \mathrm{Mpc}$ is the scale on which Hubble originally extracted his famous linear law. By conventional understanding the statistical quietness of the local Hubble flow does not make sense.

One might attempt to explain the Sandage-de Vaucouleurs paradox as a consequence of dark energy, since it is well-known that in any FLRW model which expands forever - with or without dark energy - peculiar velocities eventually decay. However, if one tries to numerically model 12.5 Gyr of motion of very local galaxies, then it turns out that the initial conditions of each galaxy appear to have the most bearing on the problem. ${ }^{19}$ In particular, one can fit a realistic motion with a cosmological constant, $\Lambda$, or alternatively in an open universe without $\Lambda$. Similar conclusions are also reached in constrained dark matter simulations of the Local Group. ${ }^{22}$ Furthermore, the $\Lambda \mathrm{CDM}$ parameters required for the velocity dispersion predicted by structure formation to match the observed velocity dispersion, do not coincide with the concordance parameters. ${ }^{23}$ Evidently a cosmological constant alone cannot explain the quietness and linearity of the local Hubble flow.

A related, but not exactly equivalent, issue is that using a conventional kinematic approach, the peculiar velocities of local galaxies appear to be at least a few factors too small to have arisen from a smooth distribution of matter at the time of recombination. ${ }^{24}$

\section{Averaging, backreaction and dark energy: the debate}

The present distribution of matter is clearly very complex, and since we cannot solve the Einstein equations for this distribution of matter analytically, there is an important question as to how we operationally match the average geometry of this distribution to the simple FLRW models that we know how to solve. Given that the nonlinear growth of structure appears to be roughly correlated to the epoch when cosmic acceleration is inferred to begin, a number of cosmologists have questioned whether the introduction of a smooth dark energy is a mistaken interpretation of the observations. ${ }^{7}$ Attention has focused on the possibility that effects attributed to cosmic acceleration may actually be due to the backreaction from the growth of inhomogeneities in determining the geometry of the observed universe, without exotic dark energy. Different interpretations of a host of complex technical issues have led to a vigorous debate. 
There are two large streams of research, which I will not focus on, for physical reasons. The first is backreaction in perturbation theory about an FLRW background, which became the focus of debate in 2005 following public attention generated by papers of Kolb and collaborators. ${ }^{25,26}$ Within perturbation theory one may demonstrate that there is a potentially significant effect from backreaction. ${ }^{26}$ However, this argument cannot be conclusive - if the second-order terms in the perturbation expansion exhibit an effect which might be interpreted as cosmic acceleration, such an effect may go away when the third-order terms are considered, and so on. Perturbation theory is very relevant near the epoch of last scattering, when the assumption of homogeneity was extremely good; but by the present epoch the nonlinear structures are so numerous and complex that we are beyond the regime of its applicability. Thus perturbation theory cannot provide a complete solution, and will not be discussed here.

The second stream of research I will not consider are those that involve exact inhomogeneous solutions of the Einstein equations, at the expense of introducing matter distributions which are unlikely. The spherically symmetric Lemaitre-Tolman-Bondi (LTB) solutions are perhaps the most wellstudied class of such models. While they may be very realistic descriptions of single voids, to apply them to the universe as a whole violates the Copernican principle, which I shall retain. One may obtain LTB solutions within which one can obtain reasonable fits to supernovae luminosity distances. ${ }^{27}$ However, in my view, given their high degree of symmetry, these are at best toy models, which one cannot hope to reproduce in structure formation scenarios based on our knowledge of the power spectrum of density perturbations at the time of last-scattering.

To confront the actual inhomogeneous universe, which has no particular spatial symmetries below the scale of homogeneity, we must deal with schemes that average the full non-linear Einstein equations. There are many schemes for constructing averages, including those of Zalaletdinov ${ }^{28}$ and Buchert. ${ }^{5}$ There are various grounds for favouring the approach of Zalaletdinov, ${ }^{28}$ which is fully covariant and averages all of the Einstein equations. However, Zalaletdinov's scheme is a general one, and for the cosmological problem additional assumptions are required. In Buchert's scheme one just average scalar quantities, and an additional integrability condition is required for the equations to close. However, with reasonable cosmological assumptions, the correlation tensor in Zalaletdinov's scheme takes the form of a spatial curvature ${ }^{29}$ and Buchert's scheme can be realized as a consistent limit. ${ }^{30}$ Furthermore Buchert's scheme yields equations which 
are close to the Friedmann equations. Given that the Friedmann equations have worked so well to date, Buchert's average would appear to give a natural framework within which corrections to the Friedmann evolution can be quantitatively examined for the universe we observe. I shall adopt Buchert's scheme, with caveats to be discussed.

Buchert's scheme strictly deals with irrotational dust cosmologies, characterized by an energy density, $\rho(t, \mathbf{x})$, expansion, $\vartheta(t, \mathbf{x})$, and shear, $\sigma(t, \mathbf{x})$, on a compact domain, $\mathcal{D}$, of a suitably defined spatial hypersurface of constant average time, $t$, and spatial 3 -metric, ${ }^{3} g_{i j}$. Angle brackets are taken to denote the spatial volume averages, e.g., for the scalar curvature

$$
\langle\mathcal{R}\rangle \equiv\left(\int_{\mathcal{D}} \mathrm{d}^{3} x \sqrt{\operatorname{det}^{3} g} \mathcal{R}(t, \mathbf{x})\right) / \mathcal{V}(t)
$$

with $\mathcal{V}(t) \equiv \int_{\mathcal{D}} \mathrm{d}^{3} x \sqrt{\operatorname{det}^{3} g}$. The important lesson of Buchert averaging is that time evolution and averaging to do not commute. ${ }^{5}$ Generally for any scalar $\Psi$

$$
\frac{\mathrm{d}}{\mathrm{d} t}\langle\Psi\rangle-\left\langle\frac{\mathrm{d} \Psi}{\mathrm{d} t}\right\rangle=\langle\Psi \vartheta\rangle-\langle\vartheta\rangle\langle\Psi\rangle
$$

The fact that the r.h.s. of (1) does not vanish, as is the case for the FLRW cosmologies, is a manifestation of backreaction.

Applied to the equations of cosmic evolution one obtains the exact Buchert equations

$$
\begin{aligned}
3 \frac{\dot{\bar{a}}^{2}}{\bar{a}^{2}} & =8 \pi G\langle\rho\rangle-\frac{1}{2}\langle\mathcal{R}\rangle-\frac{1}{2} \mathcal{Q}, \\
3 \frac{\ddot{\bar{a}}}{\bar{a}} & =-4 \pi G\langle\rho\rangle+\mathcal{Q}, \\
\partial_{t}\langle\rho\rangle & +3 \frac{\dot{\bar{a}}}{\bar{a}}\langle\rho\rangle=0,
\end{aligned}
$$

where $\bar{a}(t) \equiv\left[\mathcal{V}(t) / \mathcal{V}\left(t_{0}\right)\right]^{1 / 3}$,

$$
\mathcal{Q} \equiv \frac{2}{3}\left(\left\langle\vartheta^{2}\right\rangle-\langle\vartheta\rangle^{2}\right)-2\langle\sigma\rangle^{2}
$$

and following integrability condition follows from (2)-(5):

$$
\partial_{t}\left(\bar{a}^{6} \mathcal{Q}\right)+\bar{a}^{4} \partial_{t}\left(\bar{a}^{2}\langle\mathcal{R}\rangle\right)=0 .
$$

It is observed that the kinematic backreaction term, $\mathcal{Q}$, enters (3) with the same sign as a cosmological constant in the equivalent Raychaudhuri equation in the FLRW paradigm, but enters (2) with the opposite sign to 
a cosmological constant in the Friedmann equation. Given this fact, together with the domain-dependence of the average, the question of whether Buchert's scheme can generate sufficient backreaction to give apparent acceleration for realistic initial conditions has been the subject of much debate. On reasonable grounds one might conclude ${ }^{31}$ that while the effects are real, they are too small in magnitude to give departures sufficiently large from the FLRW expectation to register as cosmic acceleration.

It is at this point in the argument caution must be exercised. As every student of general relativity knows, one cannot simply write down a time parameter and assume that it is the parameter one measures on one's clock, without specifying how it is related to local invariants. We actually measure luminosity distances, and the deduction of cosmic acceleration involves two time derivatives. We therefore have to be extremely careful to operationally specify how $t$ is to be related to our own clocks. It must be observed that the scale-factor $\bar{a}(t)$ is not related to an exact local metric, that has been substituted in Einstein's equations. Rather we must solve the Buchert equations, and determine how the best-fit almost-FLRW geometry that is obtained relates operationally to our own measurements.

\section{Finite infinity and gravitational energy}

In a completely arbitrary inhomogeneous universe the calibration of rods and clocks at one point relative to another can vary arbitrarily. However, our clocks and rods and those of stars in distant galaxies we observe, appear to a very good approximation to be determined by geodesics of ideal solutions - the Kerr and Schwarzschild geometries - in which space is asymptotically flat, with an exact time symmetry at spatial infinity. This time symmetry, mathematically described by a timelike Killing vector, must be an idealization which breaks down at some level, since the universe is expanding.

In his pioneering work on the fitting problem, Ellis ${ }^{15}$ suggested its solution should involve the notion of finite infinity ${ }^{\mathrm{a}}$, " $f$ ", namely a timelike surface within which the dynamics of an isolated system such as the solar system can be treated without reference to the rest of the universe. After all, the matter in our galaxy and other typical galaxies broke away from the Hubble flow to be come a bound system well over 10 billion years ago. With sufficient computing resources, we can integrate the motions of stars

${ }^{a}$ As finite infinity adds a new notion of infinity to the concepts timelike, spatial and null infinity, a new mathematical symbol, $f$, is appropriate - in LATEX: $\backslash$ def $\backslash$ finfty $\{\backslash$ mathop $\{\backslash$ hbox $\{\backslash$ it fi $\}\}\}$. 
within our galaxy for billions of years without considering the dynamics of the universe outside the galaxy. Thus within finite infinity spatial geometry might be considered to be effectively asymptotically flat, and governed by "almost" Killing vectors.

The concept of finite infinity does not appear to have been further developed in the intervening two decades. However, given that the normalization of our clocks in the idealized Schwarzschild and Kerr geometries is related to the timelike Killing vector at spatial infinity, finite infinity would appear to be the appropriate reference surface for the definition of gravitational energy, which in stationary spacetimes is tied to the asymptotically flat region. ${ }^{32}$ In Newtonian terms, it is the scale at which we set the zero of the gravitational potential.

The definition of gravitational energy in general is an extremely difficult problem, on account of the fact that space itself is dynamical and can carry energy and momentum. By the strong equivalence principle, since the laws of physics must coincide with those of special relativity at a point, it is only internal energy that can be localized in an energy-momentum tensor on the r.h.s. of the Einstein equations. Any uniquely relativistic aspects of gravitational energy associated with spatial curvature and geometrodynamics cannot be included in the energy momentum tensor, but are at best described by a quasilocal formulation. ${ }^{33}$

Einstein himself worried about the problem of quasilocal gravitational energy, in terms of energy-momentum complexes, and many mathematical relativists since Einstein have also considered the problem. It is quite possible that the general problem of a definition of quasilocal energy does not have a solution, since it depends on the split of space and time. Here I will not be interested in the general problem for an arbitrary solution of the Einstein equations, but in the specific problem for a universe which was effectively homogeneous and isotropic at last scattering, with nearly scale-invariant perturbations.

The fact that quasilocal energy is not part and parcel of theoretical framework of the FLRW cosmology, is easily illustrated by the Newtonian perspective. ${ }^{34}$ The l.h.s. of the standard Friedmann equation with $\Lambda=$ 0 can be regarded as the difference of a kinetic energy density per unit rest mass, $E_{\text {kin }}=\dot{\bar{a}}^{2} /\left(2 \bar{a}^{2}\right)$ and a total energy density per unit rest mass $E_{\text {tot }}=-k /\left(2 \bar{a}^{2}\right)$ of the opposite sign to the Gaussian curvature, $k$, while the energy-momentum tensor on the r.h.s. is the Newtonian potential energy per unit rest mass. The terms in the Einstein tensor represent forms of gravitational energy, but since they are identical for all observers in an 
isotropic homogeneous geometry, one can always synchronize clocks and calibrate rods of ideal isotropic observers unambiguously.

As soon as inhomogeneity enters the game, however, one will have gradients in both the kinetic energy of expansion, and in spatial curvature. Given the present value of the Hubble constant, it is likely that the spatial curvature gradient is the most significant effect. Quasilocal energy gradients between a bound system and finite infinity could conceivably be relevant for asymptotic galactic dynamics, or even the solution of the Pioneer anomaly. This speculation is left for future work. The proposal of Refs. [3,4] is concerned principally with dynamically evolving spatial curvature gradients. In this context, one should observe that the idea that negative spatial curvature is associated with positive gravitational energy, evident already in the Newtonian framework, remains true in the LTB models, where the Gaussian curvature is replaced by an inhomogeneous energy function $E(r)$.

Cosmological gravitational energy is largely uncharted territory in the more rigorous quasilocal framework ${ }^{33}$ within which the problem should ultimately be framed. In any quasilocal formulation results depend crucially on the reference spacetime and surface of integration. Recently Chen, Liu and Nester $^{35}$ have obtained a result over which they expressed surprise, but which is consistent with the present proposal. They find that for an isotropic observer in synchronous gauge in a $k=-1$ Friedmann universe the quasilocal energy in their particular Hamiltonian formalism is negative. A similar result is obtained using a different approach by Garecki. ${ }^{36}$ These results are expected in the current approach, since one is effectively subtracting a fiducial flat spacetime in each case, and the relative sign of energy depends on the observer. An isotropic $k=0$ Friedmann observer has zero quasilocal energy in the approach of Chen, Liu and Nester; thus relative to the $k=-1$ geometry the $k=0$ geometry has negative quasilocal energy, but conversely relative to the $k=0$ geometry the $k=-1$ has positive quasilocal energy. Our viewpoint here will be that the fiducial reference point is the $k=0$ geometry of the finite infinity region. This agrees with the Newtonian version of energy in the Friedmann equation, the LTB energy function, and with the idea that binding energy is negative. ${ }^{32}$

\section{Finite infinity and the true critical density}

In the standard FLRW cosmology the critical density is proportional to the square of the Hubble parameter. However, in an inhomogeneous cosmology 
this is no longer true,

$$
\rho_{\mathrm{cr}} \neq \frac{3 H_{\mathrm{av}}^{2}}{8 \pi G}
$$

as the average Hubble parameter, $H_{\mathrm{av}}$, is related to the spatial scale of averaging and the clock chosen. In the spirit of the spherical collapse model, we are familiar with the idea that a perturbation near the time of last scattering can evolve as an FLRW model of different density, until it turns around and collapses, by which point other regions closer to the true mean density are within the past light cone.

In a universe which grew from a nearly scale-invariant spectrum of perturbations, there will always be spatial correlations of some density perturbation which is relatively far from the mean, once one samples on scales close to the spatial scale of the particle horizon. This is a simple consequence of cosmic variance, and is illustrated by the following analogy. Take a bucket filled with grains of sand of identical diameter, whose density is Gaussian distributed about a mean. Provided the container is much larger than the individual grains then the mean density of the bucket can be expected to be extremely close to the mean density of all the sand from the beach on which it was collected. If one repeats the exercise for stones of larger and larger diameter, the mean density of the bucket will on average differ more and more from the mean density of the beach as the diameter of the stones becomes comparable to the size of the bucket, by a $\sqrt{N}$ statistic.

Cosmic variance ${ }^{b}$ in the actual universe is complicated by three things. Firstly, since general relativity is causal, the bucket in the analogy is the past light cone which grows with time. Secondly, the perturbations are not uniform density but contain other perturbations embedded within them, like Russian dolls. Thirdly, below a scale of apparent homogeneity the initial perturbations can no longer be thought of as perturbations. They have undergone a non-linear transition to form the structure that we see: stars, globular clusters, and clusters of galaxies strung in filaments and bubbles around voids.

Every inflationary cosmologist who has thought about the foundations of the subject realises that the present density of the observable universe need not be the same as the whole universe, observable and unobservable, and in general these should differ on account of cosmic variance in a scale-

${ }^{\mathrm{b}} \mathrm{I}$ use the term cosmic variance in a general sense, rather than restricting it to one statistical aspect of the CMB temperature fluctuations which arise as an observable consequence of the variance in the density perturbation spectrum. 
free density perturbation spectrum. Nonetheless, in the FLRW paradigm we unwittingly end up violating this. As long as we assume that the average density evolves by the Friedmann equation, we implicitly assume that the mean density in our past light cone today is the same as the whole ensemble of observable universes. As long as we evolve the average geometry purely by the Friedmann equation, with equality in (7), we will miss an important aspect of actual cosmic evolution. Furthermore, as long we study inhomogeneity in the context of a Swiss cheese model ${ }^{37}$ by cutting and pasting holes into a background which still evolves by the Friedmann equation alone, we will still fail to uncover essential features of cosmic evolution.

There is perhaps no obvious way to define finite infinity in an arbitrary inhomogeneous background. To proceed I make the crucial observation that since our universe was effectively homogeneous and isotropic at last scattering, a notion of a universal critical density scale did exist then. It was the density required for gravity to overcome the initial uniform expansion velocity of the dust fluid. I will assume, as is consistent with primordial inflation, that the present horizon volume of the universe was very close to the critical density at last scattering, with scale-invariant perturbations.

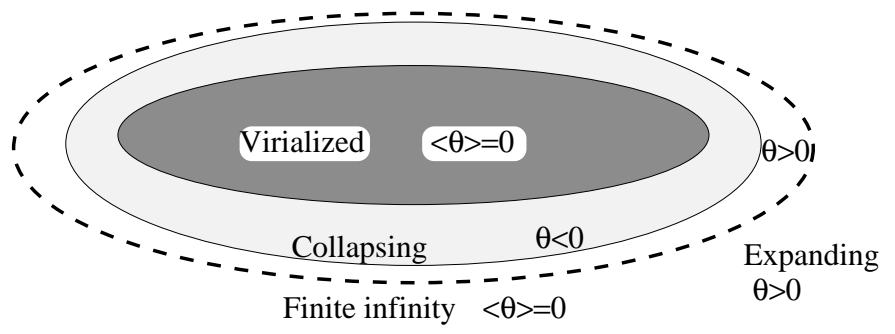

Fig. 2. Finite infinity, fi.

In view of the existence of backreaction, what is required is a notion of true critical density without assuming evolution via the Friedmann equation. This should be a dynamically evolving demarcation scale between systems which will become bound and those that are unbound, given available data within the past light cone at any epoch. As outlined in Ref. [3], and depicted in Fig. 2, finite infinity is defined in terms of a scale over which the average expansion, including virialized, collapsing and marginally expanding regions is zero, $\langle\vartheta\rangle_{f i}=0$, while being positive outside. Then $\rho_{\text {cr }} \equiv\langle\rho\rangle_{f i}$. Finite infinity is a non-static boundary analogous to the spheres cut out in the Swiss cheese model, ${ }^{37}$ but it involves average geometry rather than 
matching exact solutions, and no assumptions about homogeneity are made outside finite infinity. Finite infinity represents a physical scale expected to lie outside virialized galaxy clusters, but within the filamentary walls surrounding voids. Since it is a scale related to the true critical density, space at finite infinity boundaries can be described by the spatially flat metric

$$
\mathrm{d} s_{f i}^{2}=-\mathrm{d} \tau_{\mathrm{w}}^{2}+a_{\mathrm{w}}^{2}\left(\tau_{\mathrm{w}}\right)\left[\mathrm{d} \eta_{\mathrm{w}}^{2}+\eta_{\mathrm{w}}^{2} \mathrm{~d} \Omega^{2}\right],
$$

where $\mathrm{d} \Omega^{2} \equiv \mathrm{d} \theta^{2}+\sin ^{2} \theta \mathrm{d} \phi^{2}$. While I use the term "bubble wall" it should be recognized that an ideal bubble wall would consist of finite infinity regions touching exactly at their boundaries. Due to the existence of minivoids one should take care in identifying the scale of local walls empirically.

\section{Average homogeneity in a lumpy universe}

An important issue that has to be faced is the split of space and time. Density is not a covariant quantity, but will depend on the foliation of hypersurfaces chosen to average on, and the specification of the observers within the hypersurfaces. The hypersurfaces chosen by Buchert are the standard ones based on surfaces of constant, $t$, where this is the affine parameter on geodesics of ideal comoving observers. This choice, which is analogous to the choice of synchronous gauge in FLRW models, is always possible for an energy-momentum tensor with dust in the absence of vorticity.

While it is entirely reasonable that Buchert's choice of gauge is consistent at the volume-average position, provided one averages over suitably large spatial volumes, there is a problem of physical interpretation since observers in bound systems are located in places where the physical assumptions in Buchert's gauge choice no longer apply. As soon as regions start to collapse, geodesics will cross, vorticity comes into play and comoving coordinates cannot be chosen globally. Actual observers are in locations where the geometry is better modelled by the vacuum Schwarzschild and Kerr solutions, rather than by a rotationless dust fluid.

Since a nearly isotropic Hubble flow is observed, it is clear that at some level a uniform Hubble flow should be obtained, despite the large-scale inhomogeneity. I will make a choice of surfaces of homogeneity which implicitly solves the Sandage-de Vaucouleurs paradox by the assumption that on suitably small scales of averaging the bare Hubble flow is uniform below the scale of apparent homogeneity,

$$
\frac{1}{\ell_{r}(t)} \frac{\mathrm{d} \ell_{r}(t)}{\mathrm{d} t}=\frac{1}{3}\langle\theta\rangle_{\mathcal{D}_{1}}=\frac{1}{3}\langle\theta\rangle_{\mathcal{D}_{2}}=\cdots=\bar{H}(t),
$$


$t$ being the local proper time in each averaging region $\mathcal{D}_{I}$, and $\ell_{r} \equiv \mathcal{V}^{1 / 3}=$ $\left[\int_{\mathcal{D}_{I}} \mathrm{~d}^{3} x \sqrt{{ }^{3} g}\right]^{1 / 3}$ a relevant proper distance. Here no $\mathcal{D}_{I}$ can be contained within a finite infinity region. The important point is that although it will appear to any one observer that voids expand faster than bubble walls, the clocks of observers within voids will also tick faster than those of observers at finite infinity on account of the relative gravitational energy gradient. Thus, provided the gravitational energy and spatial curvature gradients are correlated, apparent variance in the Hubble flow for any one observer is nonetheless perfectly consistent with uniformity of the bare, or "quasilocally measurable" Hubble flow.

The uniform expansion gauge, which is equivalent to one of the standard gauges in cosmological perturbation theory, ${ }^{38}$ is given as an ansatz for defining the surfaces of homogeneity. However, it should be noted that this ansatz is less restrictive than the standard ansatz implicit in the FLRW models, where expansion is uniform, and all ideal isotropic observers also have synchronous clocks and measure the same spatial curvature on the relevant surfaces of homogeneity. Here uniformity in the bare Hubble expansion is retained, but spatial curvature and gravitational energy of isotropic observers vary in a correlated fashion.

It should be emphasized that the Copernican principle is retained here. If we compare ideal isotropic observers, some in galaxies and some in voids, they will also measure an isotropic CMB. However, they will potentially measure different mean CMB temperatures, and different angular scales in the CMB anisotropies. These two observations respectively relate to relative gravitational energy and relative spatial curvature. While the clocks of all ideal observers are synchronized initially, when the FLRW approximation was a good one, ultimately they will diverge; by $38 \%$ on average at the present epoch. Since we exchange photons with other bound systems, which are within finite infinity regions, such a large clock-rate variance is not directly detectable in experiments conceived to date.

The uniform bare Hubble constant gauge is more than simply an ansatz which implicitly solves the Sandage-de Vaucoulers paradox while dealing with the question of cosmological gravitational energy. As is argued in a separate paper ${ }^{8}$ it may be realized as a consequence of the strong equivalence principle applied to expanding space. Among the class of all possible motions of a timelike geodesic congruence there is a class of conformal motions which do not isolate any direction in space as preferred, in contrast to individual boosts. The statement then is that for such motions we cannot distinguish the circumstance in which the particles are moving from a com- 
mon origin in a static Minkowski space, or alternatively are at rest in the underlying expanding universe. The two situations are equivalent; giving a Cosmological Principle of Equivalence. ${ }^{8}$ In terms of the conceptual basis of relativistic cosmology, this amounts to a refinement of the Principle of Inertia. Not only can we not distinguish which one of Galileo's ships ${ }^{\mathrm{c}}$, or of Einstein's trains is the one that is moving; for conformal motions we can also not distinguish whether it is the collection of ships or trains that are moving, or the 'sea' or 'railyard' expanding in between.

\section{The fractal bubble model}

In Ref. [3] I have written down a Buchert average of the Einstein equations based on two scales, finite infinity regions within which the geometry is given by (8), and voids within which the geometry is negatively curved with local scale factor, $a_{\mathrm{v}}$. The geometry near the centres of voids is given by

$$
\mathrm{d} s_{\mathcal{D}_{C}}^{2}=-\mathrm{d} \tau_{\mathrm{v}}^{2}+a_{\mathrm{v}}^{2}\left(\tau_{\mathrm{v}}\right)\left[\mathrm{d} \eta_{\mathrm{v}}^{2}+\sinh ^{2}\left(\eta_{\mathrm{v}}\right) \mathrm{d} \Omega^{2}\right]
$$

The Buchert equations are not written in terms of the local geometry (8) or (10) at either finite infinity or the void centres, but in terms of an intermediate volume-average location with a volume-average time parameter, $t$, and an average scale factor, $\bar{a}$, defined by

$$
\bar{a}^{3}=f_{\mathrm{vi}} a_{\mathrm{v}}^{3}+f_{\mathrm{wi}} a_{\mathrm{w}}^{3},
$$

$f_{\mathrm{vi}}$ and $f_{\mathrm{wi}}=1-f_{\mathrm{vi}}$ being the respective initial void and wall volume fractions at last scattering. Initially $f_{\mathrm{vi}} \ll 1$, and $f_{\mathrm{wi}} \simeq 1$, since the universe is homogeneous and isotropic at last-scattering, evolving like an Einsteinde Sitter one. The Buchert average is constructed over the entire present particle horizon volume.

Although the actual surfaces of homogeneity will not coincide with Buchert's ones on small scales, we can still make use of Buchert's scheme, provided that we take care in defining the relationship between the volumeaverage quantities of Buchert's scheme, and those in a finite infinity region. Ultimately, in order to deal with actual gradients in spatial curvature between finite infinity and void centres, it may be necessary to use a scheme such as that of Zalaletdinov, ${ }^{28}$ or to look at the problem in terms of Ricci

\footnotetext{
c Actually Galileo just talked about a single ship, in a context more like Einstein's closed elevator, but the principle is the same.
} 
flow. ${ }^{39}$ This may be necessary, for example, in determining the most appropriate gauge for the background to structure formation simulations. The approach adopted in Refs. $[3,4,6]$ is that the Buchert equations are solved in volume average time, but the uniform expansion ansatz is used to calibrate observable quantities relative to observers at finite infinity. This involves a dressing of cosmological parameters over and above the volume factors considered by Buchert and Carfora. ${ }^{39,40}$

The independent Buchert equations, including the integrability condition that ensures their closure, may be written

$$
\begin{aligned}
& \bar{\Omega}_{M}+\bar{\Omega}_{k}+\bar{\Omega}_{\mathcal{Q}}=1, \\
& \bar{a}^{-6} \partial_{t}\left(\bar{\Omega}_{\mathcal{Q}} \bar{H}^{2} \bar{a}^{6}\right)+\bar{a}^{-2} \partial_{t}\left(\bar{\Omega}_{k} \bar{H}^{2} \bar{a}^{2}\right)=0,
\end{aligned}
$$

where the volume-average matter, curvature and kinematic backreaction parameters are respectively

$$
\bar{\Omega}_{M}=\frac{8 \pi G \bar{\rho}_{M 0} \bar{a}_{0}^{3}}{3 \bar{H}^{2} \bar{a}^{3}} ; \bar{\Omega}_{k}=\frac{-k_{\mathrm{v}} f_{\mathrm{vv}}{ }^{2 / 3} f_{\mathrm{v}}{ }^{1 / 3}}{\bar{a}^{2} \bar{H}^{2}} ; \bar{\Omega}_{\mathcal{Q}}=\frac{-\dot{f}_{\mathrm{v}}{ }^{2}}{9 f_{\mathrm{v}}\left(1-f_{\mathrm{v}}\right) \bar{H}^{2}} .
$$

The average curvature is due to the voids only, which are assumed to have $k_{\mathrm{v}}<0$, an overdot denotes a derivative w.r.t. volume-average time, $t$, and $\bar{H} \equiv \dot{\bar{a}} / \bar{a}$ is the volume-average or bare Hubble parameter.

Although the quantity, $\bar{H}$, has no particular significance for a general Buchert average, for our particular assumptions it represents the underlying uniform quasilocally measured Hubble flow. The quantities $\bar{\Omega}_{M}, \bar{\Omega}_{k}$ and $\bar{\Omega}_{\mathcal{Q}}$ are then interpreted as the bare cosmological parameters, relevant to a comoving isotropic observer at an average position in freely expanding space, which will be within a void, but not at its centre. These quantities are the closest analogues of the standard FLRW density parameters.

It must be recalled that the scale factor $\bar{a}$ does not correspond to an exact geometry substituted into the Einstein equations, and then solved. Rather we integrate the Buchert equations and then reconstruct the average spherically symmetric geometry

$$
\mathrm{d} s^{2}=-\mathrm{d} t^{2}+\bar{a}^{2}(t) \mathrm{d} \bar{\eta}^{2}+A(\bar{\eta}, t) \mathrm{d} \Omega^{2},
$$

the area function $A$ being defined by a horizon-volume average. ${ }^{3}$ The fact that (15) is spherically symmetric reflects the fact that the geometry is reconstructed by taking an average on our radial null geodesics. It is therefore not an LTB model, since exact spherical symmetry has not been assumed in the Einstein equations. 
Since (15) does not correspond to the local geometry of a finite infinity observer, if we assume that our rods and clocks differ little in calibration from those at finite infinity, we still have to be careful in relating our local geometry (8) to (15). We do this by conformal matching of the radial null geodesics of (8) and (15), which requires that $\mathrm{d} \eta_{\mathrm{w}}=f_{\mathrm{wi}}{ }^{1 / 3} \mathrm{~d} \bar{\eta} /\left[\bar{\gamma}\left(1-f_{\mathrm{v}}\right)^{1 / 3}\right]$. It then turns out that the finite infinity geometry may be rewritten

$$
\mathrm{d} s_{f i}^{2}=-\mathrm{d} \tau_{\mathrm{w}}^{2}+\frac{\bar{a}^{2}}{\bar{\gamma}^{2}}\left[\mathrm{~d} \bar{\eta}^{2}+r_{\mathrm{w}}^{2}\left(\bar{\eta}, \tau_{\mathrm{w}}\right) \mathrm{d} \Omega^{2}\right]
$$

where $r_{\mathrm{w}} \equiv \bar{\gamma}\left(1-f_{\mathrm{v}}\right)^{1 / 3}{f_{\mathrm{wi}}}^{-1 / 3} \eta_{\mathrm{w}}\left(\bar{\eta}, \tau_{\mathrm{w}}\right)$. The geometry (16), which now again has a more general spherically symmetric form, is effectively the closest match to the FLRW geometry we usually attempt to fit to the whole universe with the assumption that spatial curvature everywhere matches our own, and clocks everywhere are synchronized to our own wall time, $\tau_{\mathrm{w}}$.

In place of the bare cosmological parameters (14), one can define conventional dressed parameters with respect to the geometry (16), relevant to "wall observers" such as ourselves. In particular, the dressed density parameter, $\Omega_{M}$, defined according to $\Omega_{M}=\bar{\gamma}^{3} \bar{\Omega}_{M}$ is effectively the conventional parameter, whose numerical value will be similar to that which we infer in FLRW models. Since (16) is not an FLRW metric it does not make particular sense to supplement $\Omega_{M}$ by additional dressed parameters, as they will not sum to unity.

What is more significant cosmologically is that the dressed geometry (16) will yield a dressed Hubble parameter,

$$
H \equiv \frac{1}{a} \frac{\mathrm{d} a}{\mathrm{~d} \tau_{\mathrm{w}}}=\bar{\gamma} \bar{H}-\frac{\mathrm{d}}{\mathrm{d} t} \bar{\gamma}=\bar{\gamma} \bar{H}-\bar{\gamma}^{-1} \frac{\mathrm{d}}{\mathrm{d} \tau_{\mathrm{w}}} \bar{\gamma} .
$$

where $a \equiv \bar{a} / \bar{\gamma}$, which differs from the bare Hubble parameter, $\bar{H}$. Similarly, a wall observer will determine a dressed deceleration parameter $q=-\ddot{a} /\left(H^{2} a\right)$, which differs from the bare deceleration parameter $\bar{q}=$ $-\ddot{\bar{a}} /\left(\bar{H}^{2} \bar{a}\right)$.

The general solution of the two scale Buchert equations was found in Ref. [4]. The general solution is specified by four independent parameters, $\bar{H}_{0}, \bar{\Omega}_{M 0}, f_{\mathrm{v} 0}$ and $\epsilon_{i}$, which are respectively the bare Hubble constant, the bare matter density, the present epoch void volume fraction, and a small parameter $\epsilon_{i} \ll 1$ related to the initial void fraction. However, two of the four parameters are greatly restricted by taking priors ${ }^{6}$ at the surface of last scattering consistent with the CMB. Furthermore, there is a tracker solution to which all solutions with these priors approach to within $1 \%$ by 
a redshift $z \sim 37$. This effectively reduces the number of free parameters to two; we take these to be $\bar{H}_{0}$ and $f_{\mathrm{v} 0}$. The tracker solution is given by

$$
\begin{aligned}
& \bar{a}=\frac{\bar{a}_{0}\left(3 \bar{H}_{0} t\right)^{2 / 3}}{2+f_{\mathrm{v} 0}}\left[3 f_{\mathrm{v} 0} \bar{H}_{0} t+\left(1-f_{\mathrm{v} 0}\right)\left(2+f_{\mathrm{v} 0}\right)\right]^{1 / 3} \\
& f_{\mathrm{v}}=\frac{3 f_{\mathrm{v} 0} \bar{H}_{0} t}{3 f_{\mathrm{v} 0} \bar{H}_{0} t+\left(1-f_{\mathrm{v} 0}\right)\left(2+f_{\mathrm{v} 0}\right)},
\end{aligned}
$$

in terms of the volume-average cosmic time, $t$. Since the lapse function and bare Hubble parameter are related to the void fraction by $\bar{\gamma}=1+\frac{1}{2} f_{\mathrm{v}}=$ $\frac{3}{2} \bar{H} t$, the relation $\mathrm{d} \tau_{\mathrm{w}}=\bar{\gamma}^{-1} \mathrm{~d} t$ may be readily integrated to obtain the wall time, $\tau_{\mathrm{w}}$, relevant to observers in galaxies,

$$
\tau_{\mathrm{w}}=\frac{2}{3} t+\frac{2\left(1-f_{\mathrm{v} 0}\right)\left(2+f_{\mathrm{v} 0}\right)}{27 f_{\mathrm{v} 0} \bar{H}_{0}} \ln \left(1+\frac{9 f_{\mathrm{v} 0} \bar{H}_{0} t}{2\left(1-f_{\mathrm{v} 0}\right)\left(2+f_{\mathrm{v} 0}\right)}\right) .
$$

As is observed in Ref. [4], expressions for many relevant observable quantities, including effective dressed luminosity and angular diameter distances, $d_{L}$ and $d_{A}$, are readily obtained. It is particularly interesting to compare the bare volume-average tracker solution deceleration parameter,

$$
\bar{q}=\frac{2\left(1-f_{\mathrm{v}}\right)^{2}}{\left(2+f_{\mathrm{v}}\right)^{2}}
$$

with the corresponding dressed deceleration parameter

$$
q=\frac{-\left(1-f_{\mathrm{v}}\right)\left(8 f_{\mathrm{v}}^{3}+39 f_{\mathrm{v}}^{2}-12 f_{\mathrm{v}}-8\right)}{\left(4+f_{\mathrm{v}}+4 f_{\mathrm{v}}^{2}\right)^{2}},
$$

At early times, when $f_{\mathrm{v}} \ll 1$, both deceleration parameters begin at the Einstein-de Sitter value, $\bar{q} \simeq q \simeq \frac{1}{2}$. The bare deceleration parameter always remains positive, meaning that a volume-average observer in freely expanding space detects no cosmic acceleration, in accord with the intuition of the critics. ${ }^{31}$ However, the dressed deceleration parameter changes sign at epoch when $f_{\mathrm{v}} \simeq 0.5867$, at a zero of the cubic in (22), so that a wall observer does detect apparent acceleration.

The fractal bubble (FB) model provides a solution to the observational coincidence that the onset of cosmic acceleration appears to roughly coincide with the growth of large structures. Cosmic acceleration is an apparent effect that arises from gravitational energy gradients related to spatial curvature gradients. It begins when the void fraction reaches $59 \%$ at a redshift $z \simeq 0.9$. This statement itself should be able to be tested in future. Traditionally observational cosmologists define voids in terms of a density contrast threshold, when quoting void volume fractions. ${ }^{12}$ For example, one 
might argue as to whether one should take $\delta \rho / \rho<-0.9$ or some other bound. In the present model, the void volume fraction is related technically to those regions within the past light cone which are not within finite infinity. With some further refinement, it should be possible to relate this to an observational definition of void fractions. Then as surveys improve, the claims about the properties of $f_{\mathrm{v}}(z)$ can be compared with observation.

This illustrates the power of the FB model as compared to the spatially flat $\Lambda \mathrm{CDM}$ model. Both models depend on the Hubble parameter and one other free parameter. However, $\Omega_{\Lambda}$ is not directly observable, whereas the void-volume fraction, $f_{\mathrm{v}}$, is empirically observable in principle.

\section{Observational tests}

In Ref. [6] we have examined the fit of the FB model to the Riess07 gold data set $^{41}$ of SneIa. We find that with 182 data points and two degrees of freedom the best-fit $\chi^{2}=162.7$, i.e., a $\chi^{2}$ of approximately 0.9 per degree of freedom, which is a good fit. While a marginally lower $\chi^{2}=158.7$ may be obtained for the best-fit flat $\Lambda$ CDM model (with $H_{0}=62.6 \mathrm{~km} \mathrm{sec}^{-1} \mathrm{Mpc}^{-1}, \Omega_{M 0}=$ $\left.0.34, \Omega_{\Lambda 0}=0.66\right)$, the difference is not significant. In fact, a Bayesian model comparison of the FB model against a flat $\Lambda$ CDM model with priors $55 \leq H_{0} \leq 75 \mathrm{~km} \mathrm{sec}^{-1} \mathrm{Mpc}^{-1}, 0.01 \leq \Omega_{M 0} \leq 0.5$. gives a Bayes factor of $\ln B=0.27$ in favour of the FB model. Statistically, such a small margin is "not worth more than a bare mention" 42 or "inconclusive"; 43 that is to say, the two models are statistically indistinguishable.

The residual difference $\Delta \mu=\mu_{\mathrm{FB}}-\mu_{\mathrm{empty}}$, in the standard distance modulus, $\mu=5 \log _{10}\left(d_{L}\right)+25$, of the best-fit FB model from that of a coasting Milne universe of the same Hubble constant, $H_{0}=$ $61.7 \mathrm{~km} \mathrm{sec}^{-1} \mathrm{Mpc}^{-1}$, is plotted in Fig. 3 and compared with binned data from the Riess07 gold data set. Apparent acceleration occurs for positive residuals in the range, $z \lesssim 0.9$. (Note, however, that the exact range of redshifts corresponding to apparent acceleration also depends on the value of the Hubble constant of the Milne universe used to compute the residual.) The equivalent theoretical residual for the spatially flat $\Lambda \mathrm{CDM}$ model with the same values of $H_{0}$ and $\Omega_{M 0}$ is also shown. The difference in the gradients of the two curves should lead to observable differences if observing programs which are trying to measure the "equation of state, $P=w \rho$, $w(z)$, of dark energy" can achieve sufficient accuracy.

In Fig. 4 we address three major independent cosmological tests. In addition to statistical confidence limits in the $\left(H_{0}, \Omega_{M 0}\right)$ parameter space we also consider the angular scale of the sound horizon in the CMB anisotropy 


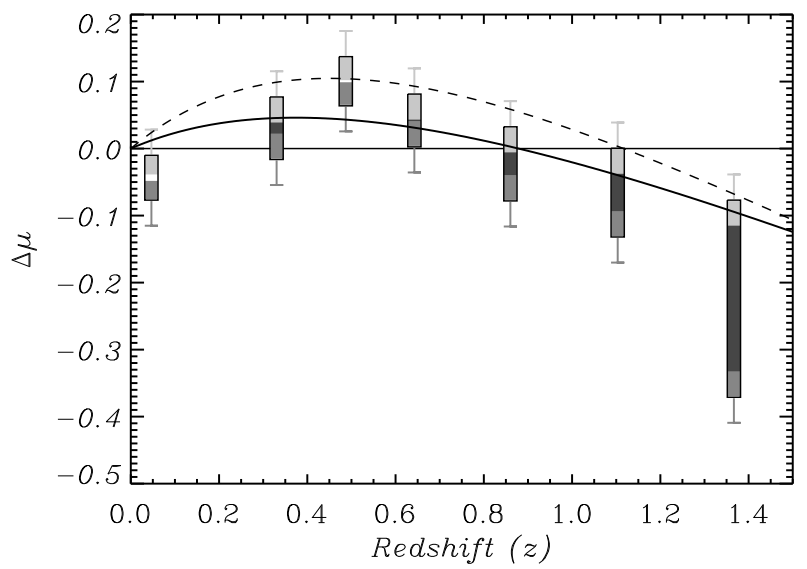

Fig. 3. The difference in the distance modulus, $\mu=5 \log _{10}\left(d_{L}\right)+25$, with $d_{L}$ in units $\mathrm{Mpc}$, of the FB model with $H_{0}=61.7 \mathrm{~km} \mathrm{sec}^{-1} \mathrm{Mpc}^{-1}, \Omega_{M 0}=0.326$ from that of an empty coasting Milne universe, with the same value of $H_{0}$. The solid curve shows the FB model expectation, and the dashed curve the expectation for a spatially flat $\Lambda$ CDM model with the same values of $H_{0}$ and $\Omega_{M 0}$. Whiskers indicate how the statistical uncertainties, shown as boxes, move when the background value of $H_{0}$ for the Milne universe which is subtracted is varied within the $2 \sigma$ limits. For further details see Ref. [6].

spectrum, ${ }^{10,11}$ and the comoving scale of the baryon acoustic oscillation (BAO) seen in galaxy clustering ${ }^{44}$ using the relevant dressed geometry (16).

Ideally we should recompute the spectrum of Doppler peaks for the FB model. However, this is a mammoth task, as the standard numerical codes have been written solely for FLRW models, and every step has to be carefully reconsidered. For this reason we first ask whether parameters exist for which the effective angular diameter scale of the sound horizon matches the angular scale of the sound horizon, $\delta=0.01 \mathrm{rad}$, of the $\Lambda \mathrm{CDM}$ model, as determined by WMAP. ${ }^{10}$ Since there is no change to the physics of recombination, but just an overall change to the calibration of cosmological parameters, this is entirely reasonable. In Fig. 4 we plot parameter ranges which match the $\delta=0.01 \mathrm{rad}$ sound horizon scale to within $2 \%, 4 \%$ and $6 \%$, using the calculation of the sound horizon given in Ref. [3], Sec. 7.2. The $2 \%$ contour would roughly correspond to the $2 \sigma$ limit if the WMAP uncertainties for the $\Lambda \mathrm{CDM}$ model are maintained. As this can only be confirmed by detailed computation of the Doppler peaks, the additional levels have been chosen cautiously.

In the case of the $\mathrm{BAO}$ scale, as we do not yet have the resources to 

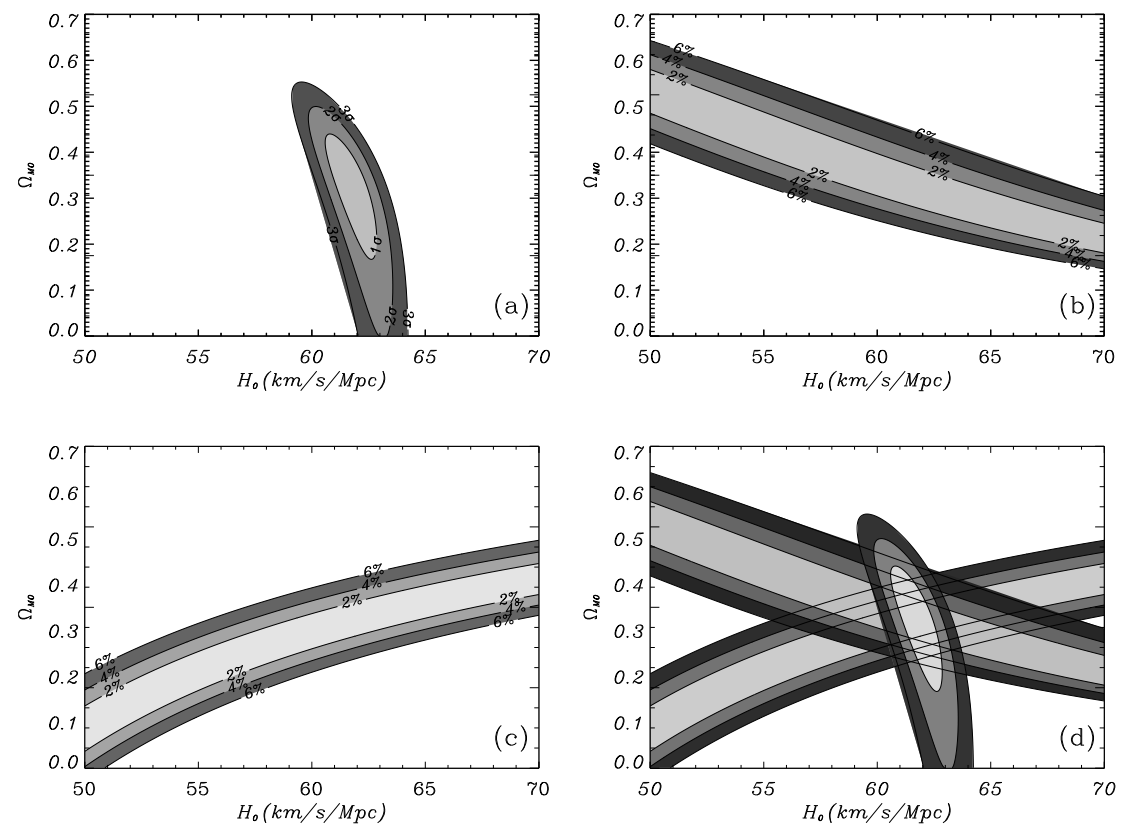

Fig. 4. Various cosmological tests in the parameter space of the dressed Hubble constant, $H_{0}$, and the dressed matter density parameter, $\Omega_{M_{0}}$. (a) Statistical confidence limits on SneIa in the Riess07 gold data set; ${ }^{41}$ (b) Parameters which match $\delta=0.01$ angular scale of sound horizon ${ }^{10,11}$ of $\Lambda \mathrm{CDM}$ model to within $2 \%, 4 \%, 6 \%$; (c) Parameters which match effective comoving $104 h^{-1}$ Mpc scale of baryon acoustic oscillation ${ }^{44}$ to within $2 \%, 4 \%, 6 \%$; (d) Overlay of panels (a),(b),(c).

analyse the galaxy clustering data directly, we also begin with a simple but effective check. Since the dressed geometry (16) does provide an effective almost-FLRW metric adapted to our clocks and rods in spatially flat regions, the effective comoving scale in this geometry should match the corresponding observed BAO scale of $104 h^{-1} \mathrm{Mpc}$. In Fig. 4 we therefore also plot parameter values which match this scale to within $2 \%, 4 \%$ or $6 \%$.

It should be noted that even if SneIa are disregarded, the parameters which fit the two independent tests relating to the sound horizon and the BAO scale agree with each other, to the accuracy shown, for values of the Hubble constant which include the value of Sandage et al. ${ }^{48}$ but not for the values of $H_{0}$ greater than $70 \mathrm{~km} \mathrm{sec}{ }^{-1} \mathrm{Mpc}^{-1}$ which best-fit the WMAP data ${ }^{10,11}$ with the FLRW model. The value $H_{0}=62.3 \pm 1.3$ (stat) \pm 5.0 (syst) $\mathrm{km} \mathrm{sec}^{-1} \mathrm{Mpc}^{-1}$ determined by the Hub- 
ble Key Team of Sandage et al. ${ }^{48}$ has been controversial, given the $14 \%$ difference from values which best-fit the WMAP data with the $\Lambda \mathrm{CDM}$ model. ${ }^{10,11}$ However, the WMAP analysis only constitutes a direct measurement of CMB temperature anisotropies; the determination of cosmological parameters involves model assumptions. In the FB model, the model assumptions are different. In particular, on account of the differences in gravitational energy and local spatial curvature between observers in bound systems, and those at the volume average in freely expanding space, the calibration of quantities related to the CMB has to be carefully reconsidered. Observers at the volume average detect a cooler mean CMB temperature ${ }^{3}$ $\bar{T}_{0}=\bar{\gamma}_{0}^{-1} T_{0}=1.98 \mathrm{~K}$ than the $T_{0}=2.73 \mathrm{~K}$ we measure. Accounting for these differences leads to concordance for parameter values which include the Hubble constant of Sandage et al. ${ }^{48}$

Table 1. Best-fit cosmological parameters derived from the independent parameters, $H_{0}, f_{\mathrm{v} 0}$.

dressed Hubble constant

present void volume fraction

mean lapse function

bare density parameter

conventional dressed density parameter mass ratio of

non-baryonic dark matter to baryonic matter

bare Hubble constant

effective dressed deceleration parameter

age of universe measured in a galaxy

$$
\begin{aligned}
& H_{0}=61.7_{-1.1}^{+1.2} \mathrm{~km} \mathrm{sec}^{-1} \mathrm{Mpc}^{-1} \\
& f_{\mathrm{v} 0}=0.76_{-0.09}^{+0.12} \\
& \bar{\gamma}_{0}=1.381_{-0.046}^{+0.061} \\
& \bar{\Omega}_{M 0}=0.125_{-0.069}^{+0.060} \\
& \Omega_{M 0}=0.33_{-0.16}^{+0.11} \\
& \left(\bar{\Omega}_{M 0}-\bar{\Omega}_{B 0}\right) / \bar{\Omega}_{B 0}=3.1_{-2.4}^{+2.5} \\
& \bar{H}_{0}=48.2_{-2.4}^{+2.0} \mathrm{~km} \mathrm{sec}^{-1} \mathrm{Mpc}^{-1} \\
& q_{0}=-0.0428_{-0.0002}^{+0.0120} \\
& \tau_{\mathrm{w} 0}=14.7_{-0.5}^{+0.7} \mathrm{Gyr} .
\end{aligned}
$$

Note: ${ }^{\text {a }} 1 \sigma$ statistical uncertainties from SneIa alone are shown.

Best-fit cosmological parameters are shown in Table 1. As statistical bounds are not available for the sound horizon and BAO tests, we show $1 \sigma$ uncertainties from SneIa only. However, given that there are many independent estimates of the dressed matter density, $\Omega_{M 0}$, we expect that the uncertainties quoted can be significantly reduced if such priors are imposed.

\subsection{Resolving the lithium abundance anomaly}

The angular scale of the sound horizon and the BAO tests have been applied assuming a volume-average baryon-to-photon ratio in the range $\eta_{B \gamma}=4.6-$ $5.6 \times 10^{-10}$ adopted by Tytler et al. ${ }^{45}$ prior to the release of WMAP1. With this range it is possible to achieve concordance with lithium abundances, 
while also better fitting helium abundances. This may resolve the primordial lithium abundance anomaly..$^{49,50}$

With the 2003 WMAP1 release, ${ }^{10}$ the baryon-to-photon ratio was increased to the very upper range of values that had previously been considered, due to its effect on the ratio of the heights of the first two Doppler peaks. This ratio of peak heights is sensitive to the mass ratio of baryons to non-baryonic dark matter - rather than directly to the baryon-to-photon ratio - as it depends physically on baryon drag in the primordial plasma. The fit to the Doppler peaks required more baryons than the range of Tytler et al. $^{45}$ admitted, when calibrated with the FLRW model. In the FB calibration, on account of the difference between the bare and dressed density parameters, a bare value of $\bar{\Omega}_{B 0} \simeq 0.03$ nonetheless corresponds to a conventional dressed value $\Omega_{B 0} \simeq 0.08$, and an overall mass ratio of baryonic matter to non-baryonic dark matter typically of about 1:3, which is larger than for $\Lambda \mathrm{CDM}$. This would certainly indicate sufficient baryon drag to accommodate the ratio of the first two peak heights.

\subsection{Spatial curvature and the ellipticity anomaly}

Since the release of the Boomerang experiment ${ }^{46}$ in 2000 , it has been generally assumed that the angular position of the first Doppler peak is a measure of the spatial curvature of the universe. However, this inference assumes that the spatial curvature is the same everywhere, as is appropriate for the FLRW models. In the present model there are spatial curvature gradients, and we must revisit the calculation from first principles, as outlined in Ref. [3]. Insofar as the angular scale of the sound horizon is reproduced for the FB calibration, the overall angular scale cannot be regarded as a measure of average spatial curvature.

If there is a sizable negative average spatial curvature at the present epoch, then there must be ways of detecting it other than via the measurement of the angular scale of the Doppler peaks. Such effects do indeed arise when one considers more subtle measurements associated with the average geodesic deviation of null geodesics. Indeed one prediction is that there should be nontrivial ellipticity in the CMB anisotropies on account of greater geodesic mixing. This effect is in fact observed, ${ }^{47}$ and is an important anomaly for the standard $\Lambda \mathrm{CDM}$ paradigm, overlooked by the majority of the community to date.

A detailed quantification of the degree of ellipticity expected, and its comparison with the observed signal, will be an important test of the FB model in future. 


\subsection{The WMAP3 normalization}

It has been recently noted that the values of the normalization of the primordial spectrum $\sigma_{8} \sim 0.76$ and matter content $\Omega_{M 0} \sim 0.24$ implied by WMAP3 are barely compatible with the abundances of massive clusters determined from X-ray measurements. ${ }^{51}$ In fact, SneIa also best fit the $\Lambda \mathrm{CDM}$ model with higher values of $\Omega_{M 0}$ comparable to the the best-fit value for the FB model, namely $\Omega_{M 0} \sim 0.33$. In the FB model the best-fit void fraction, $f_{\mathrm{v} 0} \sim 0.76$, appears to be the quantity that is mimicked by dark energy fraction, $\Omega_{\Lambda 0}$, as far as the WMAP normalization to the $\Lambda \mathrm{CDM}$ model is concerned. The flat $\Lambda \mathrm{CDM}$ model constrains $\Omega_{M 0}=1-\Omega_{\Lambda 0}$. For the FB model, the corresponding constraint, $\Omega_{M 0} \simeq \frac{1}{2}\left(1-f_{\mathrm{v} 0}\right)\left(2+f_{\mathrm{v} 0}\right)$, gives quite different larger values, consistent with many other observational determinations of the conventional matter density parameter.

\subsection{The expansion age}

Structure formation scenarios in standard $\Lambda$ CDM model have some difficulty in explaining the observed apparent very early formation of galaxies. ${ }^{52}$ Of course, structure formation contains many model-dependent assumptions, so direct measurements of the ages of metal poor stars in old globular clusters are ultimately a better indicator. The observational bounds are not very tight, because of the large uncertainties involved in particular nuclear processes. Individual ages are generally consistent with the accepted 13.7Gyr $\Lambda \mathrm{CDM}$ age of the universe, but are sometimes in tension with it, ${ }^{53}$ given our uncertainties in knowing how early on the first stars could form.

It is interesting to note that the FB model not only adds a billion years to the age of the universe as measured in a galaxy, but that the percentage difference is larger at earlier times. For concordance $\Lambda \mathrm{CDM} \tau=0.85 \mathrm{Gyr}$ at $z=6.4$ when distant quasars are seen, and $\tau=0.365$ Gyr at reionization at $z=11$. By contrast for the best-fit FB model $\tau=1.14$ Gyr at $z=6.4$ and $\tau=0.563$ Gyr at $z=11$, making the universe $34 \%$ and $54 \%$ older than concordance $\Lambda \mathrm{CDM}$ at the respective epochs.

The fact that the expansion age of the universe determined in a galaxy is greater than that of concordance $\Lambda \mathrm{CDM}$ may alleviate but not completely solve various aspects of the age problem. However, the fact that the age of the universe is $18.6 \mathrm{Gyr}$ at the volume average is a basic indicator that the whole issue of structure formation, including calibrations, needs to be re-examined from first principles. 


\subsection{The Hubble bubble}

Recent analysis of SneIa data by Jha, Riess and Kirshner ${ }^{54}$ confirms an effect which has been known about for some time, and has been interpreted as our living in a local void, ${ }^{55}$ the "Hubble bubble". If one excludes SneIa within the Hubble bubble at redshifts $z \lesssim 0.025$, then the value of the Hubble constant obtained is lower by $6.5 \pm 1.8 \%{ }^{54}$ It is for this reason that SneIa at $z \leq 0.023$ have been excluded from the Riess07 gold data set. ${ }^{41}$ The Hubble bubble is very problematic for the standard dark energy paradigm, as it can contribute a systematic error to the estimate of the equation of state parameter, $w$, which is much larger than the statistical uncertainties that precision cosmologists hope to attain. ${ }^{54,56}$

In the FB model the Hubble bubble is expected as a feature that results from the implicit resolution of the Sandage-de Vaucouleurs paradox. Since the bare Hubble parameter characterizes the uniform "quasilocally measured" Hubble flow, eq. (17) also quantifies the apparent variance in the Hubble flow below the scale of homogeneity. The present bare Hubble constant, $\bar{H}_{0}$, is lower than the global average, $H_{0}$. It represents the value we as observers in galaxies would obtain for measurements averaged solely within the plane of an ideal local bubble wall, on scales dominated by finite infinity regions. For us in particular, measurements of the Hubble constant towards the Virgo cluster would represent a scale over which we might hope to detect the bare Hubble constant, $\bar{H} \sim 48 \mathrm{~km} \mathrm{sec}^{-1} \mathrm{Mpc}^{-1}$, as this appears to be the direction that most closely represents a local bubble wall. Ultralocal minivoids ${ }^{13}$ complicate any empirical measurement.

'Local' measurements across single voids of the dominant $30 h^{-1} \mathrm{Mpc}$ diameter, ${ }^{12}$ probe the scale over which photons on null geodesics encounter the fewest finite infinity domains. Such measurements should give a Hubble 'constant' which exceeds the global average $H_{0}$ by an amount commensurate to $H_{0}-\bar{H}_{0}$. As voids are dominant by volume, an isotropic average will generally produce a Hubble 'constant' greater than $H_{0}$ for local averages until we sample on large enough scales that the volume average of walls and voids is the same as the global one. Thus the average will steadily decrease from its maximum at $\sim 30 h^{-1} \mathrm{Mpc}$ until the scale of homogeneity $\left(\sim 100 h^{-1} \mathrm{Mpc}\right)$ is reached: the Hubble bubble feature. This expectation is dramatically confirmed by the data points in Fig. 5, reproduced from a recent paper of Li and Schwarz. ${ }^{57}$ With $h=0.617$, the maximum Hubble 'constant', up to about $20 \%$ higher than average, should be attained at a scale of about $48 \mathrm{Mpc}$, thereafter steadily decreasing until leveling out at the scale of homogeneity, which must be reached before the BAO scale of 


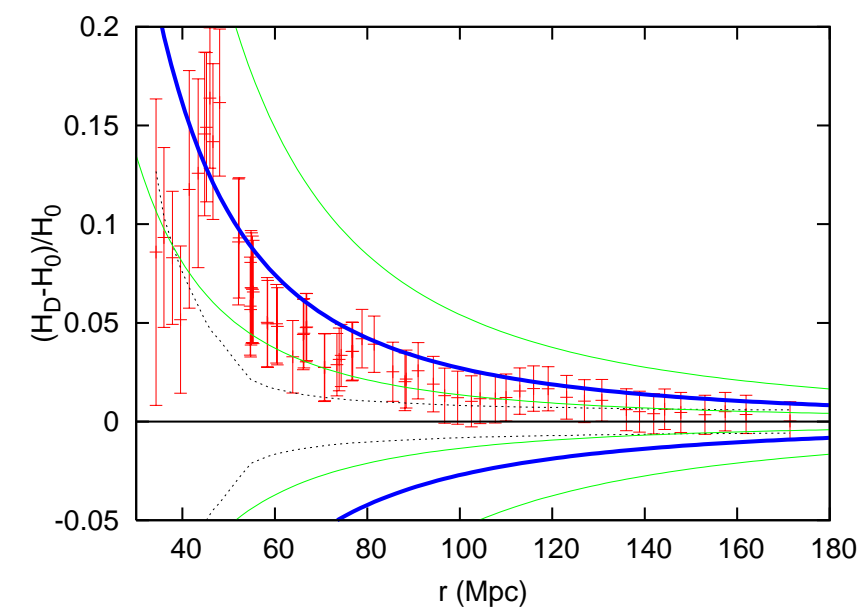

Fig. 5. Scale dependence of the normalized difference of the Hubble rate, averaged within a domain, $H_{D}$, and the global average, $H_{0}$, with data of Freedman et al. ${ }^{58}$ Courtesy of $\mathrm{Li}$ and Schwarz, ${ }^{57}$ who provide further details.

about $168 \mathrm{Mpc}$.

Fig. 5 also illustrates how the resolution of the Sandage-de Vaucouleurs paradox gives unique predictions. The two thick lines in Fig. 5 show Li and Schwarz's estimates of the bounds between which the data should lie using Buchert averaging, but not accounting for the clock effect. Given that the data indicates a consistently higher Hubble constant below the scale of homogeneity, the only explanation for the lack of great statistical scatter between the two lines - if all clocks are effectively synchronous - is that by a statistical fluke we happen to be in a large local void which is expanding faster into the surrounding medium. ${ }^{27,55}$ However, if the conventional FLRW assumptions apply beyond this Hubble bubble, then by the standard structure formation scenarios such a circumstance seems impossible.

In the FB model the Hubble bubble is not a statistical fluke, but like cosmic acceleration is an apparent effect that arises from clock rate variance. Distant observers in galaxies beyond our own Hubble bubble will also detect a Hubble bubble centred on their location. The theoretical derivation of the curve that should pass though the data points in Fig. 5 will provide an important test of the FB model. To determine this curve, and its variance, requires some knowledge of void statistics, and probably some Monte-Carlo simulations of the manner in which the scale of homogeneity is filled on average. Particular anisotropies which look like "large" voids ${ }^{14}$ might be 
expected from the variance in alignments of dominant $30 h^{-1}$ diameter voids below the scale of homogeneity. A systematic error of $1-2 \%$ in the CMB dipole subtraction ${ }^{61}$ may well be the consequence of a Rees-Sciama dipole resulting from such foreground inhomogeneities. ${ }^{62}$

While the decades long debate among astronomers about the value of the Hubble constant has been mainly dominated by arguments about systematics, ${ }^{48,59}$ the question of the scale of averaging has no doubt also played a role. Lower values of $H_{0}$ will be expected if one specifically selects directions and scales which approximate our own bubble wall. ${ }^{60}$ However, distance determinations on scales $\lesssim 50 \mathrm{Mpc}$ will generally give higher values of $H_{0}$. Since many of the first steps on the cosmic distance ladder are calibrated on such scales, many related issues require careful reconsideration.

\subsection{Prospects for future cosmological tests}

The FB model provides a strong new competitor to the standard $\Lambda$ CDM cosmology, as Table 2 shows. Indeed, since it steps out of a paradigm in which everything rests on a single equation of state, it will provide new and unique predictions. Current observational programs of course focus on the measurement of $w(z)$. The difference in expectations of the FB model for those programs will be highlighted in a forthcoming paper. ${ }^{9}$

Table 2. Model comparison

\begin{tabular}{lll}
\hline Observation & $\Lambda$ CDM & Fractal bubble model $^{\mathrm{a}}$ \\
\hline SneIa luminosity distances & Yes & Yes \\
BAO scale (clustering) & Yes & Yes \\
Sound horizon scale (CMB) & Yes & Yes \\
Doppler peak fine structure & Yes & Still to calculate \\
Integrated Sachs-Wolfe effect & Yes & Still to calculate \\
Primordial ${ }^{7}$ Li abundances & No & Yes \\
CMB ellipticity & No & [Yes] \\
CMB low multipole anomalies & No & [Foreground void(s): \\
& & Rees-Sciama dipole] \\
CMB Sunyaev-Zel'dovich signal ${ }^{63,64}$ & No & Still to calculate \\
Hubble bubble & No & Yes \\
Nucleochronology of globular clusters & Tension & Yes \\
X-ray cluster abundances & Marginal & Yes \\
Emptiness of voids & No & {$[$ Yes] } \\
Sandage-de Vaucouleurs paradox & No & Yes \\
Coincidence problem & No & Yes \\
\hline
\end{tabular}

Note: ${ }^{a}$ Square brackets indicate cases where the present indication is suggestive, but much detailed calculation remains to be done. 
The quantities that remain to be calculated in Table 2 relate largely to the CMB. Detailed construction of the Doppler peaks, to enable comparison to WMAP, and determination of parameters such as $\sigma_{8}$, is a matter of urgency. It is a rather non-trivial exercise in recalibration of standard quantities, in which all steps need to be carefully reconsidered. Detailed quantitative calculations of CMB ellipticity and the integrated Sachs-Wolfe effect can only be performed in conjunction with such an analysis.

While the calculation of the integrated Sachs-Wolfe (ISW) effect will differ in the FB model, one must be careful not to base intuition on that of perturbations on FLRW backgrounds. In particular, the observed ISW signal is believed to confirm dark energy since its consequence is a suppression of the gravitational collapse of matter at relatively recent times. ${ }^{65}$ If one replaces the words "dark energy" by "voids" in the standard qualitative explanation, then a probable description of the ISW effect in the FB model emerges. The same correlation of the ISW signal with clumped structures ${ }^{65}$ is expected; what is important is the magnitude of the effect. Mattsson ${ }^{66}$ has recently proposed an extension of the Dyer-Roeder formalism, which may have some relevance for such calculations.

In offering a new paradigm for cosmology, every observational test naturally has to be revisited from first principles. Thus there are potential tests which relate to both strong and weak gravitational lensing, for example, which have not been listed in Table 2. Qualitatively one expects voids to be emptier in the FB model than in LCDM structure formation simulations. However, the specification of the background average surfaces of homogeneity, in terms of a uniform bare Hubble expansion gauge, and a correct post-Newtonian approximation on such a background, need to be examined before structure formation simulations are attempted.

Ultimately, apparent variance in the Hubble flow below the scale of homogeneity will give predictions which cannot be reproduced in the FLRW scenario. Not only should we determine the average curve that passes through the data points in Fig. 5, we should ultimately collect thousands of SneIa measurements or other distance measurements on scales up to $200 \mathrm{Mpc}$, and test the correlation of the apparent Hubble flow with actual structure: a sort of Hubble flow tomography of the nearby universe.

\section{Conclusion}

A true "concordance cosmology" should agree with all reliable observations, and not just a carefully selected subset. A glance at Table 2 reveals that there are many anomalies in the standard $\Lambda \mathrm{CDM}$ model. Much attention 
has been focused on the CMB anisotropies, on account of the spectacular success of the WMAP mission. ${ }^{10,11}$ However, it must be recalled that many ingredients go into the analysis of the CMB temperature fluctuations. In looking at these ingredients we must ask "what is the weakest link"?

In my view, the weakest link is not primordial nucleosynthesis, ${ }^{49}$ which is based on nuclear physics that we understand very well, but the cosmological model. The weakest link comes from abandoning the theoretical principles that we are careful to apply in other circumstances. In particular, in general relativity one should model the universe with the matter distribution one observes, rather than trying to impose onto the universe a simple mathematical solution based on some simplifying symmetry.

It is unfortunate that general relativists have been obsessed by exact solutions of Einstein's equations, whether they involve likely or unlikely approximations for the matter distribution. We should face up to the fact that the solution for the actual matter distribution is analytically intractable, and therefore the question of cosmological averaging $5,7,15,16,28-30$ is paramount. Furthermore, once we do take an average we must address the fundamental problem that the relationship of rods and clocks at one point to those at a distant point, a conceptual centrepiece of general relativity, is highly non-trivial once gradients in spatial curvature and gravitational energy are considered. In an expanding universe these involve subtle dynamical aspects of general relativity, which cannot be localized at a point on account of the equivalence principle.

Some colleagues when presented with Table 2, and the fact that the right hand column is based entirely on general relativity with no new or exotic physics (beyond a need for non-baryonic dark matter), suggest I should invoke Ockham's Razor at this point and declare the cosmological constant dead. Other colleagues, who sometimes confess to Newtonian intuition, are of the view that a clock-rate difference of $38 \%$ accumulated between bound systems and the volume average over the lifetime of the universe is nonetheless so great that I must be mistaken somewhere, in spite of Table 2.

In my view caution should always be exercised, but this includes caution with the conceptual basis of our theory and the operational interpretation of measurements. To those who are uncomfortable with my proposal about cosmological quasilocal gravitational energy let me ask the following: Without reference to an asymptotically flat static reference scale, which does not exist given the universe is expanding, and without reference to a background which evolves by the Friedmann equation at some level, ${ }^{37}$ an assumption 
which is manifestly violated by the observed inhomogeneities, What keeps clocks synchronized in cosmic evolution? Please explain.

In the FB proposal the fact that our FLRW approximation has served so well is understood as a consequence of the fact that the bare Hubble flow is uniform, despite large-scale inhomogeneities. Uniformity of the quasilocally measured Hubble flow does not imply uniformity of spatial curvature, nor of gravitational energy, however. If we examine the Hubble flow over scales on which the average gradients are not large, we will not see large statistical scatter in the Hubble flow. But averaged over larger scales, below the scale of homogeneity, we will see a variance in the apparent Hubble flow, that agrees with observations of the statistical properties of voids, ${ }^{12}$ and which seems to accord with Fig. 5. The fact that there is a statistically dominant void scale ${ }^{12}$ may well be associated with the evolution of the scale corresponding to the second (rarefaction) Doppler peak in the CMB anisotropy spectrum, just as evolution of the first (compression) peak appears to statistically define the cutoff for the scale of homogeneity.

Any proposal which seeks to genuinely shift the paradigm against accepted intuition, even a conservative proposal based on a principled approach $^{3,8}$ to our best theory of gravitation, naturally faces much initial scepticism. Nonetheless, I suspect that in future cosmologists will wonder how we ever could have expected that two decades of precision measurements would simply confirm the naïve cosmological models constructed in the 1920 s, long before we knew what the universe actually looked like. I suspect that the coming decades will see us explore and finally understand the arcane territory of general relativity relating to quasilocal gravitational energy, ${ }^{32,33,35,36}$ informed directly by new cosmological observations.

Acknowledgements I am grateful to many people for discussions and correspondence including, in particular, Thomas Buchert. I also thank Anthony Fairall and Dominik Schwarz for granting me permission to reproduce their Figures 1 and 5 respectively.

\section{References}

1. R. Durrer and R. Maartens, arXiv:0711.0077.

2. P.J.E. Peebles, Astrophys. J. 557, 495 (2001).

3. D.L. Wiltshire, New J. Phys. 9, 377 (2007).

4. D.L. Wiltshire, Phys. Rev. Lett. 99, 251101 (2007).

5. T. Buchert, Gen. Relativ. Grav. 32, 105 (2000); Gen. Relativ. Grav. 33, 1381 (2001).

6. B.M. Leith, S.C.C. Ng and D.L. Wiltshire, Astrophys. J. 672, L91 (2008). 
7. S. Räsänen, JCAP 11, 003 (2006); M.N. Célérier, New Adv. Phys. 1 (2007) 29 [astro-ph/0702416]; N. Li and D.J. Schwarz, Phys. Rev. D 76, 083011 (2007); T. Buchert, arXiv:0707.2153; and references therein.

8. D.L. Wiltshire, "Cosmological principle of equivalence", in preparation.

9. D.L. Wiltshire, "Observational quantities in the fractal bubble universe", in preparation.

10. C.L. Bennett et al., Astrophys. J. Suppl. 148, 1 (2003).

11. D.N. Spergel et al., Astrophys. J. Suppl. 170, 377 (2007).

12. F. Hoyle and M.S. Vogeley, Astrophys. J. 566, 641 (2002); Astrophys. J. 607, 751 (2004).

13. A.V. Tikhonov and I.D. Karachentsev, Astrophys. J. 653, 969 (2006).

14. L. Rudnick, S. Brown and L.R. Williams, arXiv:0704.0908.

15. G.F.R. Ellis, in B. Bertotti, F. de Felice and A. Pascolini (eds), General Relativity and Gravitation, (Reidel, Dordrecht, 1984) pp. 215-288.

16. G.F.R. Ellis and W. Stoeger, Class. Quantum Grav. 4, 1697 (1987).

17. F. Sylos Labini, M. Montuori and L. Pietronero, Phys. Rep. 293, 61 (1998).

18. Y. Baryshev, AIP Conf. Proc. 822, 23 (2006).

19. A.D. Chernin, I.D. Karachentsev, M.J. Valtonen, V.P. Dolgachev, L.M. Domozhilova and D.I. Makarov, Astron. Astrophys. 415, 19 (2004).

20. A. Sandage, G.A. Tammann and E. Hardy, Astrophys. J. 172, 253 (1972).

21. G. de Vaucouleurs, Science 167, 1203 (1970).

22. Y. Hoffman, L.A. Martinez-Vaquero, G. Yepes and S. Gottlöber, arxiv:0711.4989.

23. M. Axenides and L. Perivolaropoulos, Phys. Rev. D 65, 127301 (2002).

24. A.B. Whiting, Astron. J. 131, 1996 (2006).

25. E.W. Kolb, S. Matarrese, A. Notari and A. Riotto, hep-th/0503117.

26. E.W. Kolb, S. Matarrese and A. Riotto, New J. Phys. 8, 322 (2006).

27. M.N. Célérier, Astron. Astrophys. 353, 63 (2000); J.W. Moffat, JCAP 05, 001 (2006); H. Alnes, M. Amarzguioui and O. Gron, Phys. Rev. D 73, 083519 (2006); T. Biswas, R. Mansouri and A. Notari, astro-ph/0606703.

28. R.M. Zalaletdinov, Gen. Relativ. Grav. 24, 1015 (1992); 25, 673 (1993).

29. A.A. Coley, N. Pelavas and R.M. Zalaletdinov, Phys. Rev. Lett. 95, 151102 (2005); A.A. Coley and N. Pelavas, Phys. Rev. D 74, 087301 (2006); D 75, 043506 (2007).

30. A. Paranjape and T.P. Singh, Phys. Rev. D 76, 044006 (2007).

31. A. Ishibashi and R.M. Wald, Class. Quantum Grav. 23, 235 (2006).

32. J. Katz, Class. Quantum Grav. 22, 5169 (2005); J. Katz, D. Lynden-Bell and J. Bičák, Class. Quantum Grav. 23, 7111 (2006); D. Lynden-Bell, J. Katz and J. Bičák, Phys. Rev. D 75, 024040 (2007); Err. ibid. D 75, 044901 (2007).

33. L.B. Szabados, Living Rev. Rel. 7, 4 (2004).

34. H. Bondi, "Cosmology", (Cambridge Univ. Press, 1960)

35. C.M. Chen, J.L. Liu and J.M. Nester, Mod. Phys. Lett. A 22, 2039 (2007).

36. J. Garecki, arXiv:0708.2783.

37. A. Einstein and E.G. Straus, Rev. Mod. Phys. 17, 120 (1945); Err. ibid. 18, 148 (1946).

38. J.M. Bardeen, Phys. Rev. D 22, 1882 (1980). 
39. T. Buchert and M. Carfora, Class. Quantum Grav. 19, 6109 (2002).

40. T. Buchert and M. Carfora, Phys. Rev. Lett. 90, 031101 (2003).

41. A.G. Riess et al., Astrophys. J. 659, 98.

42. R.E. Kass and A.E. Raftery, J. Am. Statist. Assoc. 90, 773 (1995).

43. R. Trotta, Mon. Not. R. Astr. Soc. 378, 72 (2007).

44. D.J. Eisenstein et al., Astrophys. J. 633 (2005) 560; S. Cole et al., Mon. Not. R. Astr. Soc. 362 (2005) 505.

45. D. Tytler, J.M. O'Meara, N. Suzuki, and D. Lubin, Phys. Scripta T85, 12 (2000).

46. P. de Bernardis et al. [Boomerang Collaboration], Nature 404 (2000) 955.

47. V.G. Gurzadyan, C.L. Bianco, A.L. Kashin, H. Kuloghlian and G. Yegorian, Phys. Lett. A 363 (2007) 121; V.G. Gurzadyan, P. de Bernardis, G. De Troia, C.L. Bianco, A.L. Kashin, H. Kuloghlian, S. Masi, F. Piacentini, G. Polenta and G. Yegorian, Mod. Phys. Lett. A 20 (2005) 813.

48. A. Sandage, G.A. Tammann, A. Saha, B. Reindl, F.D. Macchetto and N. Panagia, Astrophys. J. 653 (2006) 843.

49. G. Steigman, Int. J. Mod. Phys. E 15 (2006) 1.

50. M. Asplund, D.L. Lambert, P.E. Nissen, F. Primas and V.V. Smith, Astrophys. J. 644 (2006) 229.

51. G. Yepes, R. Sevilla, S. Gottloeber, and J. Silk, Astrophys. J. 666, L61 (2007).

52. K. Glazebrook et al., Nature 430, 181 (2004); A. Cimatti et al., Nature 430, 184 (2004).

53. L.M. Krauss and B. Chaboyer, Science 299, 65 (2003).

54. S. Jha, A.G. Riess and R.P. Kirshner, Astrophys. J. 659, 122 (2007).

55. K. Tomita, Mon. Not. R. Astr. Soc. 326, 287 (2001); Prog. Theor. Phys. 106, 929 (2001).

56. W.M. Wood-Vasey et al., Astrophys. J. 666, 694 (2007).

57. N. Li and D.J. Schwarz, arXiv:0710.5073.

58. W.L. Freedman et al., Astrophys. J. 553, 47 (2001).

59. F. van Leeuwen, M. W. Feast, P. A. Whitelock and C. D. Laney, Mon. Not. R. Astr. Soc. 379, 723 (2007).

60. M. Federspiel, G.A. Tammann and A. Sandage, Astrophys. J. 495, 115 (1998).

61. P.E. Freeman, C.R. Genovese, C.J. Miller, R.C. Nichol and L. Wasserman, Astrophys. J. 638 (2006) 1.

62. A. Rakic, S. Räsänen and D.J. Schwarz, Mon. Not. R. Astr. Soc. 369 (2006) L27.

63. R. Lieu, J.P.D. Mittaz and S.-N. Zhang, Astrophys. J. 648, 176 (2006).

64. R.M. Bielby and T. Shanks, Mon. Not. R. Astr. Soc. 382, 1196 (2007).

65. S. Boughn and R. Crittenden, Nature 427, 45 (2004).

66. T. Mattsson, arXiv:0711.4264. 